\title{
Satisfacción con la Vida y la Autoeficacia Específica para el Afrontamiento del Estrés, en los Estudiantes Universitarios de la Escuela de Psicología de la Universidad Privada César Vallejo de Lima Sur, 2017
}

\author{
Dr. Guillermo Pastor Morales Romero \\ gmorales@une.edu.pe \\ https://orcid.org/0000-0002-5686-7661 \\ Universidad Nacional de Educación Enrique Guzmán y Valle. Lima - Perú.

\begin{abstract}
Pedro Lacho Quispe pedrolachoqf@gmail.com https://orcid.org/0000-0001-8769-0988 Universidad Nacional de Educación Enrique Guzmán y Valle. Lima - Perú.
\end{abstract}

Dr. Oscar Eugenio Pujay Cristobal opujay@undac.edu.pe https://orcid.org/0000-0002-2051-9403 Universidad Nacional Daniel Alcides Carrión. Cerro Pasco - Perú.

Dr. César Gerardo León Velarde cesoar@hotmail.com https://orcid.org/0000-0002-8273-1995 Universidad Tecnológica del Perú. Lima - Perú.

\author{
Jorge Augusto Sanchez Ayte \\ jsanchez@untels.edu.pe \\ https://orcid.org/0000-0001-9734-3381 \\ Universidad Nacional Tecnológica de Lima Sur \\ Mg. Yanet Rosario Paucar Manrique \\ yanetpaucarmanrique@gmail.com \\ https://orcid.org/0000-0001-9275-5547 \\ Universidad Nacional Federico Villarreal. Lima - Perú. \\ Mg. Sofía Gamarra Mendoza \\ sofia.gamarra@epg.usil.pe \\ https://orcid.org/0000-0002-2297-3503 \\ Universidad San Ignacio de Loyola. Lima - Perú.
}

\section{RESUMEN}

El objetivo general fue establecer la relación entre satisfacción con la vida y la autoeficacia específica para el afrontamiento del estrés en los estudiantes universitarios de Psicología de la Universidad Privada César Vallejo. La investigación de enfoque 
cuantitativo y no experimental, tipo correlacional, corte descriptivo. La población son los estudiantes de psicología de la Universidad Privada César Vallejo. Muestra conformada por 80 estudiantes universitarios del IX ciclo turno noche. La técnica empleada son psicosométricas, y la encuesta. Se organizo tablas y figuras para la estadística descriptiva, en la estadística inferencias se utilizó la prueba de normalidad y correlación de Pearson. Los resultados muestran que los valores de sig $<0.05$, por lo tanto, se rechaza H0, se acepta que los datos de la muestra no provienen de una distribución normal, por lo tanto, para probar las hipótesis planteadas se usó la prueba de Kolmogorov-Smirnov y el coeficiente de Pearson, se rechaza la hipótesis nula y se admite la hipótesis del $\mathrm{H}_{\mathrm{G}}$. Satisfacción con la vida se relaciona significativamente con la autoeficacia específica para el afrontamiento del estrés, en los estudiantes universitarios de Psicología de la Universidad Privada César Vallejo, es correlación positiva fuerte, de acuerdo a los índices de correlación.

Palabras claves: Satisfacción con la vida; autoeficacia; afrontamiento del estrés; estudiantes universitarios; psicología. 


\title{
Satisfaction with Life and Specific Self-Efficacy for Coping with Stress, in University Students of the School of Psychology of the César Vallejo Private University of Lima Sur, 2017
}

\begin{abstract}
The general objective was to establish the relationship between life satisfaction and specific self-efficacy for coping with stress in psychology university students at the César Vallejo Private University. Research with a quantitative and non-experimental approach, correlational type, descriptive cut. The population is psychology students from the César Vallejo Private University. Sample made up of 80 university students from the IX night shift cycle. The technique used are psychosometric, and the survey. Tables and figures were organized for descriptive statistics, Pearson's normality and correlation test was used for statistical inferences. The results show that the values of sig $<0.05$, therefore, $\mathrm{H} 0$ is rejected, it is accepted that the sample data do not come from a normal distribution, therefore, to test the hypotheses, the Kolmogorov test was used- Smirnov and Pearson's coefficient, the null hypothesis is rejected and the HG hypothesis is admitted. Satisfaction with life is significantly related to specific self-efficacy for coping with stress, in psychology university students of the César Vallejo Private University, it is a strong positive correlation, according to the correlation indexes.
\end{abstract}

Keywords: Satisfaction with life; self-efficacy; coping with stress; University students; psychology.

Artículo recibido: 05 de Abril 2021 Aceptado para publicación: 28 de Mayo 2021

Correspondencia: gmorales@une.edu.pe Conflictos de Interés: Ninguna que declarar 


\section{INTRODUCCIÓN}

El estilo de vida que tienen los estudiantes, la dinámica familiar que experimentan, el tipo de crianza recibido, el colegio donde han estudiado, los intereses, la personalidad, las formas de solucionar los problemas, las expectativas y preocupaciones respecto al futuro, el tipo de economía que manejan de alguna manera se refleja en su desenvolvimiento personal, es decir, en las diferentes dimensiones de su vida y a su vez en su salud mental. Es por ello importante estudiar y proteger la calidad de vida de nuestros jóvenes, objeto de estudio para que se desempeñen adecuadamente como estudiantes y sean exitosos como profesionales y como seres humanos para que en un futuro próximo sean padres de familia satisfechos y sean buenos modelos para sus hijos de la nueva sociedad que nos espera. En cuanto al afrontamiento del estrés es prioritario resaltar que los jóvenes aprendan a desarrollar el autocontrol y manejo de emociones impulsando el conocimiento y práctica de los mecanismos positivos para afrontar estas situaciones de tensión evitando conflictos y relaciones interpersonales poco saludables.

El desarrollo de la autoeficacia, depende del vínculo inicial que se tuvo y en base a ello, se forman los recursos personales (Armus, Duhalde, Oliver y Woscoboinik, 2012). De igual manera, Repetur (2005) enfatiza en la relación cálida entre el niño y la madre como crucial para el desarrollo saludable del menor, lo que tendrá un efecto en las relaciones sociales, lazos emocionales, regulación de estados emocionales, percepción y manejo del propio sujeto sobre sus problemas (Zulueta, 2005 y Armus, Duhalde, Oliver y Woscoboinik, 2012).

El trabajo describe el planteamiento del problema, en ella se preguntó ¿Cuál es la relación entre satisfacción con la vida y la autoeficacia específica para el afrontamiento del estrés, en los estudiantes universitarios de la Escuela de Psicología de la Universidad Privada César Vallejo de Lima Sur?; del mismo modo se definieron los objetivos, importancia, alcance y limitaciones que se tuvieron para realizar esta investigación. Se explica en el marco teórico: antecedentes, bases teóricas de ambas variables y la definición de términos básicos. También las hipótesis, variables y su operacionalización, la metodología que se usó para generar esta investigación: enfoque, tipo, diseño, población, muestra, técnicas, instrumentos, y en la parte ultima se muestra los resultados de la investigación, su análisis, discusión, para terminar en las conclusiones y recomendaciones. 


\section{ESTRATEGIAS METODOLÓGICAS O MATERIALES Y MÉTODOS}

\section{Enfoque de investigación:}

La presente investigación se encuentra como una investigación dentro del enfoque cuantitativo y no experimental, de acuerdo con Hernández, Fernández y Baptista (2010). Este enfoque de investigación estudia los fenómenos sin intervención del investigador, es decir, se observa el fenómeno tal cual y se analiza a través de métodos estadísticos.

\section{Tipo de investigación:}

La investigación que realizada se encuentra dentro del tipo de investigación correlacional, "este tipo de estudios tiene como finalidad conocer la relación o grado de asociación que exista entre dos o más conceptos, categorías o variables en un contexto en particular" (Hernández \& Baptista, 2010). Esta investigación plantea conocer la posible relación que existe entre la satisfacción con la vida y la autoeficacia específica para el afrontamiento del estrés en los estudiantes de la Escuela de Psicología de la Universidad César Vallejo de Lima Sur.

\section{Diseño de investigación:}

El presente estudio es de un diseño descriptivo-correlacional que nos permite en un solo momento recoger la información de las variables estudiadas en los mismos sujetos, con el objeto de identificar la intensidad de la relación entre estas variables. (Hernández, Fernández \& Baptista, 2010).

El diagrama representativo del diseño correlacional es el siguiente:

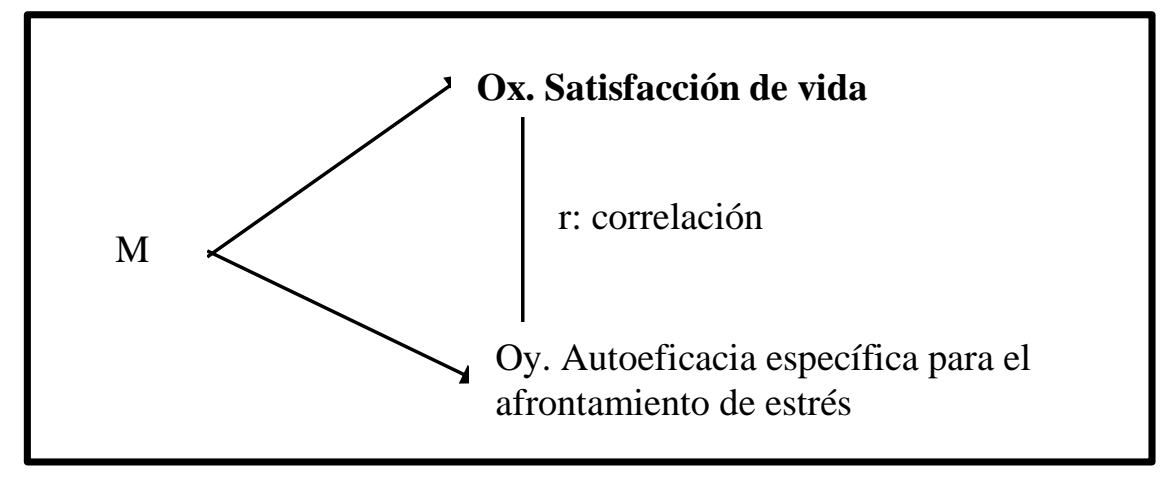

Figura 1.- Diseño de investigación

M: Muestra de investigación

$\mathrm{O}_{\mathrm{X}}, \mathrm{O}_{\mathrm{Y}}$ : Observación de las variables, satisfacción con la vida y autoeficacia para el afrontamiento de estrés

r: Relación de las variables 


\section{Método.}

- La metodología utilizada para la recolección de datos fue transversal, se usó las escalas validadas para su aplicación.

\section{Población y muestra:}

\section{Población:}

- Para los fines de la presente investigación, la población estuvo conformada por los estudiantes universitarios del IX ciclo turno noche de la carrera de psicología de la Universidad Privada César Vallejo sede San Juan de Lurigancho.

\section{Muestra:}

- La muestra de estudio es una muestra censal que consta de 80 estudiantes universitarios del IX ciclo turno noche de la carrera de psicología de la Universidad Privada César Vallejo sede San Juan de Lurigancho. La muestra se considera censal porque se seleccionó el $100 \%$ de la población al considerarla un número manejable de sujetos. En este sentido, Ramírez (1997) establece que la muestra censal es aquella donde todas las unidades de investigación son consideradas como muestra.

\section{Técnicas e instrumentos de recolección de información:}

Se utilizará las siguientes técnicas

- Técnicas psicométricas: escalas validadas de satisfacción con la vida y autoeficacia para el afrontamiento del estrés.

- Análisis de documentos: de fuentes primarias, y secundarias para la elaboración de marco teórico.

\section{Instrumentos:}

\section{Escala de satisfacción con la vida.}

La satisfacción vital se define como una valoración global que la persona hace sobre su vida, comparando lo que ha conseguido, sus logros, con lo que esperaba obtener, sus expectativas (Diener et al., 1985; Pavot, Diener, Colvin y Sandvik, 1991). Basándose en esta definición teórica y puesto que los instrumentos que se habían desarrollado hasta el momento, o constaban de un único ítem, o sólo eran apropiados para poblaciones de la tercera edad, o incluían otros factores además de la satisfacción con la vida.

El objetivo de la escala es evaluar los aspectos cognitivos del bienestar. El modelo usado para la medición es la versión en castellano de la SWLS de Diener \& Griffin (1985) escala que se puede utilizar con jóvenes y adultos. 
Diener et al. (1985) crearon una escala multi-ítem para medir la satisfacción vital. El instrumento resultante mostró buenas propiedades psicométricas: Alfa de Cronbach de 0,87, correlación test-retest de 0,82 a los dos meses y correlaciones ítem-test entre 0,57 y 0,75. El análisis factorial extrajo un único factor que explicaba el $66 \%$ de la varianza.

Para obtener la puntuación total de la escala de Diener, debe sumarse la puntuación de cada una de las cinco afirmaciones, obteniéndose un índice de felicidad subjetiva en función de los siguientes tramos:

$35-31$ pts. Muy satisfecho

$26-30$ pts. Satisfecho

21-25 pts. Algo satisfecho

20 pts. Neutral

15-19 pts. Algo insatisfecho

10-14 pts. Insatisfecho

$5-9$ pts. Muy insatisfecho

\section{Escala de autoeficacia.}

La escala de autoeficacia específica para el afrontamiento del estrés (EAEAE; Es un instrumento que está validado y se han usado en numerosas investigaciones a lo largo de todo el mundo y en Sudamérica y en nuestro país aún no se han realizado muchos estudios, en la Universidad Nacional de Educación Enrique Guzmán y Valle, existe un trabajo relacionado con aprendizaje y autoeficacia (Suárez, 2016).

La escala de AEAE (Godoy\& Vásquez). Este instrumento está compuesto por 8 ítems, 4 de los cuales (ítems 2, 4, 5, 7) evalúan el componente de expectativas de eficacia (EE) del constructo de autoeficacia. Los 4 restantes (ítems $1,3,6,8)$ el de expectativas de resultado (ER).

Los ítems están formulados tanto en sentido directo $(2,3,5,7)$ como en sentido inverso $(1,4,6,8)$.

Las respuestas se presentan en formato tipo Likert con 5 alternativas desde "completamente en desacuerdo" a "completamente de acuerdo", con valores de 1 a 5.

Se obtiene también una puntuación total (Total) resultado de la suma de las dos puntuaciones parciales. La escala mide la autoeficacia de afrontamiento al estrés de una forma directa, correspondiendo a puntuaciones más elevadas una mayor confianza en los 
recursos personales para el manejo del estrés. Todos los participantes contestarán la EAEAE.

\section{RESULTADOS Y DISCUSIÓN}

Tabla 1: Baremos de comparación.

Variable X. Satisfacción con la vida.

\begin{tabular}{|c|c|c|c|c|c|c|c|}
\hline $\begin{array}{c}\text { Nivel } \\
\text { variable o } \\
\text { dimensión }\end{array}$ & $\begin{array}{c}\text { Muy } \\
\text { insatisfecho }\end{array}$ & Insatisfecho & $\begin{array}{c}\text { Algo } \\
\text { insatisfecho }\end{array}$ & Neutro & $\begin{array}{c}\text { Algo } \\
\text { satisfecho }\end{array}$ & Satisfecho & $\begin{array}{c}\text { Muy } \\
\text { satisfecho }\end{array}$ \\
\hline $\begin{array}{l}\text { Satisfacción } \\
\text { con la vida }\end{array}$ & $5-9$ & $10-14$ & $15-19$ & 20 & $21-25$ & $26-30$ & $31-35$ \\
\hline $\begin{array}{l}\text { Item } \mathrm{N}^{\circ} \\
\text { Item } \mathrm{N}^{\circ} 1\end{array}$ & - & - & $1,2,3$ & - & 4,5 & - & 6,7 \\
\hline Item $\mathrm{N}^{\circ} 2$ & - & - & $1,2,3$ & - & 4,5 & - & 6,7 \\
\hline Item $N^{\circ} 3$ & - & - & $1,2,3$ & - & 4,5 & - & 6,7 \\
\hline Item $\mathrm{N}^{\circ} 4$ & - & - & $1,2,3$ & - & 4,5 & - & 6,7 \\
\hline Item $N^{\circ} 5$ & - & - & $1,2,3$ & - & 4,5 & - & 6,7 \\
\hline
\end{tabular}

Tabla 2: Tabla de frecuencia variable satisfacción con la vida.

\begin{tabular}{lcccc} 
& Frecuencia & Porcentaje & $\begin{array}{c}\text { Porcentaje } \\
\text { válido }\end{array}$ & $\begin{array}{c}\text { Porcentaje } \\
\text { acumulado }\end{array}$ \\
\hline Algo insatisfecho & 1 & 1 & 1 & 1 \\
Algo satisfecho & 59 & 74 & 74 & 75 \\
Neutral & 10 & 13 & 13 & 88 \\
Satisfecho & 10 & 12 & 12 & 100 \\
Total & 80 & 100 & 100 & \\
\hline
\end{tabular}

\section{Interpretación.}

1. El $74 \%$ de los estudiantes universitarios de la Escuela de Psicología de la Universidad Privada César Vallejo de Lima Sur, referencian estar algo satisfechos con la vida, pues tienen algunas áreas vitales en las que desearían una mejora.

2. El 13\% de los estudiantes universitarios de la Escuela de Psicología de la Universidad Privada César Vallejo de Lima Sur, referencian opinión neutral con respecto a la satisfacción con la vida.

3. El 12\% de los estudiantes universitarios de la Escuela de Psicología de la Universidad Privada César Vallejo de Lima Sur, referencian estar satisfechos con la vida, sienten que la mayoría de las cosas de la vida son en general buenas.

4. El 1\% de los estudiantes universitarios de la Escuela de Psicología de la Universidad Privada César Vallejo de Lima Sur, referencian estar algo insatisfechos con la vida, 
pues tienen problemas que son pequeños pero significativos en varias áreas de sus vidas.

Tabla 3. Ítem $N^{\circ}$ 2: Las condiciones de mi vida son excelentes.

\begin{tabular}{lcccc} 
& Frecuencia & Porcentaje & $\begin{array}{c}\text { Porcentaje } \\
\text { válido }\end{array}$ & $\begin{array}{c}\text { Porcentaje } \\
\text { acumulado }\end{array}$ \\
\hline Algo insatisfecho & 13 & 16 & 16 & 16 \\
Algo satisfecho & 53 & 66 & 66 & 83 \\
Muy satisfecho & 14 & 18 & 18 & 100 \\
Total & 80 & 100 & 100 & \\
\hline
\end{tabular}

\section{Interpretación.}

1. El 66\% de los estudiantes universitarios de la Escuela de Psicología de la Universidad Privada César Vallejo de Lima Sur, referencian estar algo satisfechos con la vida, ya que las condiciones de sus vidas son excelentes.

2. El $18 \%$ de los estudiantes universitarios de la Escuela de Psicología de la Universidad Privada César Vallejo de Lima Sur, referencian estar muy satisfechos con la vida, ya que las condiciones de sus vidas son excelentes.

3. El 16\% de los estudiantes universitarios de la Escuela de Psicología de la Universidad Privada César Vallejo de Lima Sur, referencian estar algo insatisfechos con la vida, ya que las condiciones de sus vidas no son excelentes.

Tabla 4. Ítem $N^{\circ}$ 3: Estoy satisfecho(a) con mi vida.

\begin{tabular}{lcccc}
\hline & Frecuencia & Porcentaje & $\begin{array}{c}\text { Porcentaje } \\
\text { válido }\end{array}$ & $\begin{array}{c}\text { Porcentaje } \\
\text { acumulado }\end{array}$ \\
\hline Algo insatisfecho & 16 & 20 & 20 & 20 \\
Algo satisfecho & 53 & 66 & 66 & 86 \\
Muy satisfecho & 11 & 14 & 14 & 100 \\
Total & 80 & 100 & 100 & \\
\hline
\end{tabular}

\section{Interpretación.}

1. El $66 \%$ de los estudiantes universitarios de la Escuela de Psicología de la Universidad Privada César Vallejo de Lima Sur, referencian estar algo satisfechos con la vida.

2. El 14\% de los estudiantes universitarios de la Escuela de Psicología de la Universidad Privada César Vallejo de Lima Sur, referencian estar muy satisfechos con la vida.

3. El 20\% de los estudiantes universitarios de la Escuela de Psicología de la Universidad Privada César Vallejo de Lima Sur, referencian estar algo insatisfechos con la vida. 
Tabla 5. Ítem $\mathrm{N}^{\circ}$ 4: Hasta ahora, he tenido las cosas importantes que deseo en la vida.

\begin{tabular}{lcccc}
\hline & Frecuencia & Porcentaje & $\begin{array}{c}\text { Porcentaje } \\
\text { válido }\end{array}$ & $\begin{array}{c}\text { Porcentaje } \\
\text { acumulado }\end{array}$ \\
\hline Algo insatisfecho & 5 & 6 & 6 & 6 \\
Algo satisfecho & 61 & 76 & 76 & 83 \\
Muy satisfecho & 14 & 18 & 18 & 100 \\
Total & 80 & 100 & 100 & \\
\hline
\end{tabular}

\section{Interpretación.}

1. El 76\% de los estudiantes universitarios de la Escuela de Psicología de la Universidad Privada César Vallejo de Lima Sur, referencian estar algo satisfechos con la vida, ya que, hasta ahora, han tenido las cosas importantes que desean en la vida.

2. El $18 \%$ de los estudiantes universitarios de la Escuela de Psicología de la Universidad Privada César Vallejo de Lima Sur, referencian estar muy satisfechos con la vida, ya que, hasta ahora, han tenido las cosas importantes que desean en la vida.

3. El 6\% de los estudiantes universitarios de la Escuela de Psicología de la Universidad Privada César Vallejo de Lima Sur, referencian estar algo insatisfechos con la vida, ya que, hasta ahora, no han tenido las cosas importantes que desean en la vida.

Tabla 6. Ítem $N^{\circ}$ 5: Si pudiese vivir mi vida de nuevo, no cambiaría casi nada.

\begin{tabular}{lcccc} 
& Frecuencia & Porcentaje & $\begin{array}{c}\text { Porcentaje } \\
\text { válido }\end{array}$ & $\begin{array}{c}\text { Porcentaje } \\
\text { acumulado }\end{array}$ \\
\hline Algo insatisfecho & 8 & 10 & 10 & 10 \\
Algo satisfecho & 63 & 79 & 79 & 89 \\
Muy satisfecho & 9 & 11 & 11 & 100 \\
Total & 80 & 100 & 100 & \\
\hline
\end{tabular}

\section{Interpretación.}

1. El 79\% de los estudiantes universitarios de la Escuela de Psicología de la Universidad Privada César Vallejo de Lima Sur, referencian estar algo satisfechos con la vida, ya que, si pudiesen vivir su vida de nuevo, no cambiaría casi nada.

2. El 11\% de los estudiantes universitarios de la Escuela de Psicología de la Universidad Privada César Vallejo de Lima Sur, referencian estar muy satisfechos con la vida, ya que, si pudiesen vivir su vida de nuevo, no cambiaría casi nada. 
3. El 10\% de los estudiantes universitarios de la Escuela de Psicología de la Universidad Privada César Vallejo de Lima Sur, referencian estar algo insatisfechos con la vida, ya que, si pudiesen vivir su vida de nuevo, harían cambios.

Tabla 7. Baremos de comparación.

Variable Y. Autoeficacia específica para el afrontamiento del estrés.

\begin{tabular}{lccc}
\hline \multicolumn{1}{c}{ Nivel variable o dimensión } & Bajo & Medio & Alto \\
\hline Autoeficacia específica para el & $8-19$ & $20-30$ & $31-40$ \\
afrontamiento del estrés & $4-9$ & $10-15$ & $16-20$ \\
Eficacia & $4-9$ & $10-15$ & $16-20$ \\
Resultado & 1,2 & 3 & 4,5 \\
Ítem $\mathrm{N}^{\circ} 1$ & 1,2 & 3 & 4,5 \\
Ítem $\mathrm{N}^{\circ} 2$ & 1,2 & 3 & 4,5 \\
Ítem $\mathrm{N}^{\circ} 3$ & 1,2 & 3 & 4,5 \\
Ítem $\mathrm{N}^{\circ} 4$ & 1,2 & 3 & 4,5 \\
Ítem $\mathrm{N}^{\circ} 5$ & 1,2 & 3 & 4,5 \\
Ítem $\mathrm{N}^{\circ} 6$ & 1,2 & 3 & 4,5 \\
Ítem $\mathrm{N}^{\circ} 7$ & 1,2 & 3 & 4,5 \\
Ítem $\mathrm{N}^{\circ} 8$ & & & \\
\hline
\end{tabular}

Tabla8. Variable Y. Autoeficacia específica para el afrontamiento del estrés.

\begin{tabular}{lcccc}
\hline & Frecuencia & Porcentaje & $\begin{array}{c}\text { Porcentaje } \\
\text { válido }\end{array}$ & $\begin{array}{c}\text { Porcentaje } \\
\text { acumulado }\end{array}$ \\
\hline Alto & 3 & 4 & 4 & 4 \\
Medio & 77 & 96 & 96 & 100 \\
Total & 80 & 100 & 100 & \\
\hline
\end{tabular}

1. El $96 \%$ de los estudiantes universitarios de la Escuela de Psicología de la Universidad Privada César Vallejo de Lima Sur, referencian un nivel medio de autoeficacia específica para el afrontamiento del estrés, ya que cuentan con mayor confianza en los propios recursos personales para el manejo del estrés.

2. El 4\% de los estudiantes universitarios de la Escuela de Psicología de la Universidad Privada César Vallejo de Lima Sur, referencian un nivel alto de autoeficacia específica para el afrontamiento del estrés, ya que cuentan con mayor confianza en los propios recursos personales para el manejo del estrés. 


\section{Prueba de hipótesis.}

\section{Hipótesis general.}

La satisfacción con la vida se relaciona significativamente con la autoeficacia específica para el afrontamiento del estrés, en los estudiantes universitarios de la Escuela de Psicología de la Universidad Privada César Vallejo de Lima Sur. Se quiere determinar la relación entre la satisfacción con la vida y la autoeficacia específica para el afrontamiento del estrés.

Para ello analizaremos el tipo de distribución que presentan los datos en cada variable, a través de la prueba de Kolmogorov. Considerando el valor de la prueba, se determinará el uso del estadístico de correlación: si los datos presentan distribución normal, se utilizará el paramétrico (Pearson), caso contrario, no paramétrico (Rho de Spearman).

\section{Prueba de normalidad: Kolmogorov.}

Es una prueba de bondad de ajuste, permite determinar si los datos provienen de una población con distribución normal.

\section{- Planteamiento de hipótesis:}

Ho: los datos presentan distribución normal

Ha: los datos no presentan distribución normal

- Nivel de significancia:

$\alpha=0.05$

- Prueba:

Las puntaciones de satisfacción con la vida y autoeficacia específica para el afrontamiento del estrés se han ingresado al programa estadístico SPSS, y se ha realizado la prueba de Kolmogorow, obteniendo el siguiente resultado:

Tabla9. Prueba de Kolmogorov-Smirnov para una muestra.

\begin{tabular}{lccc}
\hline & & Satisfacción & Autoeficacia \\
\hline Parámetros normales(a, b) & Media & 80 & 80 \\
& Desviación & 22.88 & 25.91 \\
& típica & 2.319 & 2.189 \\
Diferencias más extremas & Absoluta & .141 & .197 \\
& Positiva & .141 & .197 \\
& Negativa & -.095 & -.104 \\
Z de Kolmogorov-Smirnov & & 1.261 & 1.758 \\
Sig. asintót. (bilateral) & & .083 & .061 \\
\hline
\end{tabular}


a) La distribución de contraste es la Normal.

b) Se han calculado a partir de los datos.

- Regla de decisión:

$\checkmark \mathrm{Si}$ “p “ (sig) $<0.05$, se rechaza la $\mathrm{H}_{\mathrm{G} 0}$.

$\checkmark \mathrm{Si}$ “p " $(\operatorname{sig})>0.05$, se acepta la HEO.

\section{- Decisión:}

De acuerdo a los resultados obtenidos en la prueba de Kolmogorow para la variable satisfacción con la vida, el valor de $\mathrm{p}=0.083$ (sig bilateral), dicho resultado es mayor a 0.05 , por lo tanto, los datos presentan distribución normal. De acuerdo a los resultados obtenidos en la prueba de Kolmogorow para variable autoeficacia específica para el afrontamiento del estrés, el valor de $\mathrm{p}=0.061$ (sig bilateral), dicho resultado es mayor a 0.05 , por lo tanto, los datos presentan distribución normal.

Las pruebas de hipótesis se han trabajado con el estadístico de correlación $\mathrm{r}$ de Pearson, puesto que los datos de las variables cumplen el supuesto de normalidad.

\section{Coeficiente de correlación de Pearson (r).}

El coeficiente de correlación de Pearson (r), es un método de correlación para variables medidas por intervalos o razón y para relaciones lineales. Se calcula a partir de las puntuaciones obtenidas en una muestra con dos variables. Se relacionan las puntuaciones obtenidas de una variable con las puntuaciones obtenidas de la otra, con los mismos participantes o casos.

Formula de Pearson:

$$
r=\frac{n\left(\sum x y\right)-\left(\sum x\right)\left(\sum y\right)}{\sqrt{\left[n\left(\sum x^{2}\right)-\left(\sum x\right)^{2}\right]\left[n\left(\sum y^{2}\right)-\left(\sum y\right)^{2}\right]}}
$$

El coeficiente $r$ de Pearson puede variar de $-1.0 \mathrm{a}+1.0$.

$$
-1 \leq r \leq+1
$$


Tabla10. Tabla de Valoración de Pearson.

Dónde:

\begin{tabular}{ll} 
Coeficiente & \multicolumn{1}{c}{ Tipo de correlación } \\
-1.00 & Correlación negativa perfecta \\
-0.90 a -0.99 & Correlación negativa muy fuerte \\
-0.70 a -0.89 & Correlación negativa fuerte \\
-0.40 a -0.69 & Correlación negativa media o moderada \\
-0.20 a -0.39 & Correlación negativa débil \\
-0.01 a -0.19 & Correlación negativa muy débil \\
0.00 & No existe correlación alguna entre las variables \\
0.01 a 0.19 & Correlación positiva muy débil \\
0.20 a 0.39 & Correlación positiva débil \\
0.40 a 0.69 & Correlación positiva media o moderada \\
0.70 a 0.89 & Correlación positiva fuerte \\
0.90 a 0.99 & Correlación positiva muy fuerte \\
+1.00 & Correlación positiva perfecta
\end{tabular}

\section{Prueba de hipótesis}

- Hipótesis estadísticas:

$\checkmark$ Hipótesis nula (Ho): $\rho=0$, La satisfacción con la vida no se relaciona con la autoeficacia específica para el afrontamiento del estrés, en los estudiantes universitarios de la Escuela de Psicología de la Universidad Privada César Vallejo de Lima Sur.

$\checkmark$ Hipótesis alterna (Ha): $\boldsymbol{\rho} \neq \mathbf{0}$, La satisfacción con la vida se relaciona significativamente con la autoeficacia específica para el afrontamiento del estrés, en los estudiantes universitarios de la Escuela de Psicología de la Universidad Privada César Vallejo de Lima Sur.

- Nivel de Significancia: $=\mathbf{0 . 0 5}$

- Diagrama de dispersión:

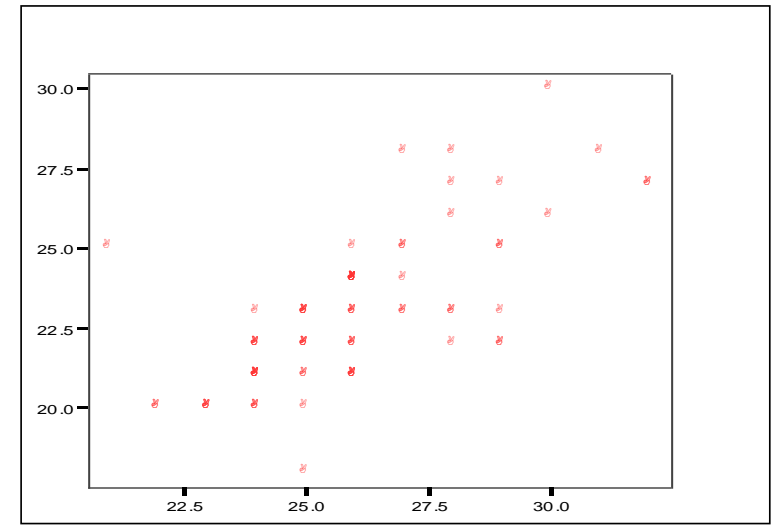

Figura 2.- Diagrama de dispersión 1. 
- Estadístico de la prueba:

Los puntajes obtenidos en cada variable han sido ingresados al programa estadístico SPSS, obteniendo el siguiente resultado:

Tabla 11. Correlación de Pearson (r).

\begin{tabular}{llcc}
\hline & & Satisfacción & Autoeficacia \\
\hline Satisfacción & Correlación de Pearson & 1 & $0,704(* *)$ \\
& Sig. (bilateral) & & .000 \\
& $\mathrm{~N}$ & 80 & 80 \\
\multirow{3}{*}{ Autoeficacia } & Correlación de Pearson & $0,704(* *)$ & 1 \\
& Sig. (bilateral) & .000 & \\
& $\mathrm{~N}$ & 80 & 80 \\
\hline
\end{tabular}

** La correlación es significativa al nivel 0,01 (bilateral).

- Decisión

$\checkmark \mathrm{Si}$ "p” (sig.) $<0.05$, se rechaza la Ho

$\checkmark$ Si "p" (sig.) $>0.05$, se acepta la Ho

El valor de $\mathrm{p}=0.000$ es menor a 0.05 . Por lo tanto se rechaza la hipótesis nula.

\section{- Conclusión}

De acuerdo a la tabla mostrada, el coeficiente de correlación de Pearson $r=0,704$. Entonces la satisfacción con la vida se relaciona significativamente con la autoeficacia específica para el afrontamiento del estrés, en los estudiantes universitarios de la Escuela de Psicología de la Universidad Privada César Vallejo de Lima Sur, según el cuadro de Sampieri es correlación positiva fuerte, de acuerdo a los índices de correlación.

\section{CONCLUSIÓN O CONSIDERACIONES FINALES}

Primera.- Se concluye que, la satisfacción con la vida se relaciona significativamente con la autoeficacia específica para el afrontamiento del estrés, en los estudiantes universitarios de la Escuela de Psicología de la Universidad Privada César Vallejo de Lima Sur, el 74\% de los estudiantes, referencian estar algo satisfechos con la vida, pues tienen algunas áreas vitales en las que desearían una mejora y el $96 \%$ referencian un nivel medio de autoeficacia específica para el afrontamiento del estrés, ya que cuentan con mayor confianza en los propios recursos personales para el manejo del estrés. 
Segunda.- Se concluye que, la satisfacción con la vida se relaciona significativamente con la expectativa de eficacia, estudiantes universitarios de la Escuela de Psicología de la Universidad Privada César Vallejo de Lima Sur, el 98\% de los estudiantes referencian un nivel medio de autoeficacia específica para el afrontamiento del estrés-expectativas de eficacia, ya que el afrontamiento del estrés se refiere a los juicios subjetivos (creencias) sobre las capacidades personales de control del estrés.

Tercera.- Se concluye que, la satisfacción con la vida se relaciona significativamente con la expectativa de resultado, en los estudiantes universitarios de la Escuela de Psicología de la Universidad Privada César Vallejo de Lima Sur, el 95\% de los estudiantes referencian un nivel medio de autoeficacia específica para el afrontamiento del estrés-expectativas de resultados, ya que se refieren a las creencias personales sobre las consecuencias de las capacidades personales (o carencia de ellas) para manejar el estrés.

\section{LISTA DE REFERENCIAS}

Atienza, F., Pons, D., Balaguer, I., y García Merita, M. (2000). Propiedades Psicométricas de la Escala de Satisfacción con la Vida en Adolescentes. Psicothema, 12, 2, 314-319. Recuperado de: http://www.psicothema.com/psicothema.asp?id=296.

Bandura A \& Walters R. (1990). Aprendizaje social y desarrollo de la personalidad. Editorial: Madrid: Alianza Universidad.

Bandura, A. (1986). Social foundations of thought and action: A social cognitive theory. Englewood Cliffs, NJ: Prentice-Hall.

Bandura, A. (1994). Self-Efficacy. In V. S. Ramachaudran (Ed.), Encyclopedia of (Vol. 4, Pp. 71-81). New York: Academic Press. (Reprinted In H. Friedman [Ed.], Encyclopedia Of Mental Health. San Diego: Academic Press, 1998). Recuperado de: https://www.uky.edu/ eushe2/bandura/bandura1994ehb.pdf

Bandura, A. (2001). Social cognitive theory: An agentic perspective. Annual review of psychology, 52(1), 1-26. Recuperado de: https://scholar.google.com.pe/scholar?cluster=17874734641974926521\&hl=es\& as_sdt $=2005 \&$ sciodt $=0,5$. 
Becerra, S. (2013). Rol de estrés percibido y su afrontamiento en las conductas de salud de estudiantes universitarios de Lima. (Tesis de licenciatura inédita).Pontificia Universidad Católica del Perú, Lima, Perú.

Becerra, S. (2016). Descripción de las conductas de salud en un grupo de estudiantes universitario de Lima. Pontificia Universidad Católica del Perú. Lima, Perú. Revista de psicología 34(2), 240-260.

Casas, F. (1991). El concepto de calidad de vida en la intervención social en el ámbito de la infancia, en Colegio Oficial de Psicólogos. (Ed.), III Jornadas de Psicología de la Intervención Social (pp. 649-672). Madrid: Instituto Nacional de Servicios Sociales.

Castro, A. (2009). El bienestar psicológico: cuatro décadas de progreso: http://aufop.com/aufop/uploaded_files/articulos/1258587233.pdf.

Cornejo, M. (2005). Comparación Social y bienestar subjetivo entre estudiantes de una Universidad privada de Lima. Tesis: PUCP.

Condori, L. (2013). Relación de: la autoeficacia ante el estrés, personalidad, percepción de bienestar psicológico y de la salud con el rendimiento académico en universitarios. (Tesis doctoral, universidad nacional mayor de san marcos, lima).

Diener, E. \& Diener, M. (1995). Cross Cultural Correlates of life satisfaction and selfesteem. Journal of Personality and Social Psychology, 68 (4),653-663.

Diener, E. (1984). Subjective Well-Being. Psychological Bulletin, 3(8), 67-113.

Diener, E., Oishi \& lucas R. Personality, culture, and subjective well-being: Emotional and Cognitive Evaluations of Life. Annu. ev. Psychol. 2003. 54: 403-25.

Diener. E., \& Griffin. S. (1985). The satisfaction with Life scale. Jornal of Personality Assessment, 49, 71-75.

Folkman, S., \& DeLongis, A. (1986). Appraisal, coping, health status, and psychological symptoms. Journal of personality and social psychology, 50(3), 571.

Forsythe, C. \& Compas, B. Cogn Ther Res (1987) 11: 473. Recuperado de: https://link.springer.com/article/10.1007/BF01175357.

Galvanovskis, A. \& Sosa, A.D. (2000). Calidad de vida en el periodo de jubilación. En La Psicología Social en México. Vol. VIII (pp. 39-45). México: Asociación Mexicana de Psicología Social. Disponible en: http://www.amepso.org/revistas.php?memstatus=2. 
Gimeno X, (2004). Satisfacción en el trabajo de los directores de las escuelas secundarias públicas de la región de jacobina. (Tesis doctoral, programa de doctoral calidad y procesos de innovación educativa. Departamento de pedagogía aplicada universidad autónoma de Barcelona).

Godoy D., \& León, R. (2004a). Propiedades psicométricas de la « Escala de Autoeficacia Específica para el Afrontamiento del Estrés» I: Fiabilidad. $2^{\circ}$ Congreso HispanoPortugués de Psicología, Lisboa (Portugal).

Hernández, R., Fernández, C. \& Baptista, P. (2010). Metodología de la Investigación. (5 ed.) México: Mc Graw-Hill Interamericana Editores.

Laca A, \& Muñiz, J.; (2005). Satisfacción con la vida de algunos colectivos mexicanos: una discusión sobre la psicología del bienestar subjetivo. Enseñanza e Investigación en Psicología, julio-diciembre, 325-336.

Mauricio, H. (2016). La influencia de la satisfacción con la familia y el estatus material con el desempeño. (Tesis para optar el título de licencia do en psicología con mención en psicología social, pontifica universidad católica de lima). Recuperado de http://tesis.pucp.edu.pe/repositorio/handle/123456789/7938.

Mikkelsen, F. (2009, 6 abril). Satisfacción con la vida y las estrategias de afrontamiento en un grupo de adolescentes universitario de Lima. (Tesis de licenciatura, pontifica universidad católica de lima). Recuperado de http://tesis.pucp.edu.pe/repositorio/handle/123456789/23/browse?value=Mikkels en+Ramella\%2C+Flor+de+Mar\%C3\%ADa\&type=author.

Montero R. (2017). El Perú mejora en 12 indicadores de desarrollo. Diario del bicentenario el peruano. Recuperado de. http://www.elperuano.pe/noticia-elperu-mejora-12-indicadores-desarrollo-56365.aspx.

Moyano, N. \& Muñoz, M. (2013). Propiedades Psicométricas de la Escala de Satisfacción con la Vida de Diener. Revista Argentina de Clínica Psicológica, XXII Agosto-, 161-168. Recuperado de: http://www.redalyc.org/comocitar.oa?id=281931436008.

Murillo, G. (2013). Autoeficacia y felicidad en ingresantes de una universidad privada de Trujillo. Revista de Psicología Trujillo Perú 15(1):22-36,68-81, 2013 Recuperado de http://revistas.ucv.edu.pe/index.php/R_PSI/article/download/209/118. 
Navarro, L. (2013).Dos aproximaciones complementarias para medir satisfacción con la vida en una comunidad al sur de Perú. Tesis: PUCP.

Ortega-Álvarez, F., Núñez-Hergueta, E., Molero, D. y Torres-González, J.A. (2015). Diversidad emocional y satisfacción vital en futuros docentes. Revista nacional e internacional de educación inclusiva, 8(3), 205-217.

Organización mundial de la salud. (1997). Programme on mental health division of mental health and prevention of substance abuse world health organization. Recuperado de. http://www.who.int/mental_health/media/68.pdf.

Pavot, W, Diener, E. (1993). The affective and cognitive context of self-reported measures of subjective well-being. Soc Indic Res ,Volumen 28, numero 1 pp 1-20. Recuperado de: https://link.springer.com/article/10.1007\%2FBF01086714.

Pavot, W., Diener, E.D., Colvin, C.R. y Sandvik, E. (1991). Further validation of the Satisfaction With Life Scale: Evidence for the cross-method convergence of wellbeing. Journal of Personality Assessment, 57, 149-161.

Rosales, M. (2017). Autoeficacia y felicidad en estudiantes universitarios de la carrera de psicología de una universidad privada de lima sur. (Tesis para optar el título de Licenciado en Psicología con mención en Psicología, Universidad Autónoma de Perú) Lima.

Rozalén M. (2014). Creencias De Autoeficacia y Coaching. Como Mejorar La Productividad de las personas. IV Jornadas Internacionales Mentoring \& Coaching: Universidad - Empresa (23, 24 y 25 noviembre, Madrid) Págs. 22 - 45 http://innovacioneducativa.upm.es/jimcue_09/comunicaciones/02_2245_Coaching_Creencias.pdf.

Santrock, J. (2014). Psicología de la Educación. Ed: Mc Graw Hill. Interamericana editores. México, D.F.

Schunk, D. H. (2012). Teorías del aprendizaje. Una perspectiva educativa. Pearson Educación, estado de México.

Seligman, M. (2006). Authentic Hapiness. Ediciones B,S.A Barcelona España

Selye H. (1950). The physiology and pathology of exposure to stress, a treatise based on the concepts of the general-adaptationsyndrome and the diseases of adaptation. Montreal: ACTA, Inc., Medical Publishers. Recuperado de: https://link.springer.com/article/10.1007/BF01175357. 
Suarez, C. (2016). Concepciones de aprendizaje y autoeficacia académica en los estudiantes de estudios generales de la universidad san Martin de Porres. (Tesis para optar el título de Maestría en Docencia Universitaria Universidad Enrique Guzmán y Valle Escuela de Posgrado sección Maestría) Perú - Lima.

Tejero, C. \& Ruiz, R. (2016) Satisfacción con la vida y autoeficacia en jugadores de baloncesto en silla de ruedas. Revista de Psicología del Deporte. 2016. Vol. 25, núm. 1, pp. 51-56. Recuperado de http://www.rpd-online.com/article/view/v25n1-tejero-gonzalez-de-etal.

Vásquez, L. (2009). Eficacia de un programa de entrenamiento en autoeficacia específica para el afrontamiento de estrés en pacientes psicóticos. (Tesis doctoral de la Universidad de Granda Facultad de Psicóloga, Departamento de Personalidad, Evaluación y Tratamiento Psicológico). Recuperado de https://hera.ugr.es/tesisugr/18139784.pdf.

Veenhoven, R. (1994). El estudio de la satisfacción con la vida. Intervención Psicosocial, 3, 87-116. Recuperado de: http://hdl.handle.net/1765/16195.

Woolfolk, A. (2010). Perspectivas Cognitivas Sociales del Aprendizaje y la Motivación. En Psicología Educativa. 11a. Edición Pearson Educación,.(p.348-356) Impreso En México. 